7th International Workshop on Astronomy and

Relativistic Astrophysics (IWARA 2016)

International Journal of Modern Physics: Conference Series

Vol. 45 (2017) 1760063 (4 pages)

(C) The Author(s)

DOI: $10.1142 / \mathrm{S} 2010194517600631$

\title{
Diffractive Production of Quarkonium in p-A Collisions at LHC
}

\author{
Érison S. Rocha and Magno V. T. Machado \\ Instituto de Física \\ Universidade Federal do Rio Grande do Sul (UFRGS) \\ Av. Bento Gonçalves 9500, 91501-970 Porto Alegre, RS, Brazil \\ erison.rocha@ufrgs.br,magno.machado@ufrgs.br
}

Published 15 August 2017

\begin{abstract}
In the present work, we investigated the exclusive diffractive production of charmonium in pronton-nucleus collisions at the Large Hadron Collider (LHC) energies. Such exclusive production reactions possess a cleaner experimental signal than inclusive production once it is characterized by low multiplicity of particles between final produced state and the incident protons of collider beam. The considered theoretical framework was the perturbative model of Quantum Cromodynamics (QCD) for the Pomeron exchange. In particular, we have used the resolved Pomeron model which depends on the Pomeron flux and Pomeron parton distribution functions. The main goal is to provide predictions for the single diffractive cross section for the J/Psi meson production at the energies of $5.02 \mathrm{TeV}$ and $8.8 \mathrm{TeV}$. We have shown that a measurement is feasible as the order of magnitude is 400 microbarns even absorption corrections. Furthermore, we analyze the ratio of the diffractive to inclusive cross section which allows theoretical uncertainties to be canceled, mostly those associated to approximations performed in inclusive case.
\end{abstract}

Keywords: Diffractive, Charmonium LHC.

PACS numbers: 13.87.Ce

\section{Introduction}

In this work we investigate the phenomenological approaches of diffractive exclusive production of quarkonia in collisions proton-nucleus for the $\mathrm{LHC}^{\mathrm{a}}$ energies. These bound states are called quarkonium in analogy with positronium states in electromagnetic interactions. Such production of charm-anticharm states, called exclusive, possesses an experimental signal more "clean" than inclusive production because it is characterized by low multiplicity of particles between final produced states and the incident protons of the collider beam ${ }^{1}$. This is an open topic in literature and theoretical predictions in energies window available data at LHC are crucial.

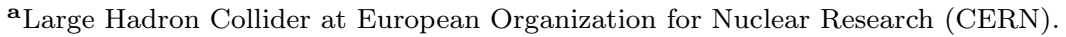

This is an Open Access article published by World Scientific Publishing Company. It is distributed under the terms of the Creative Commons Attribution 4.0 (CC-BY) License. Further distribution of this work is permitted, provided the original work is properly cited. 


\section{E. S. Rocha \& M. V. T. Machado}

\section{Theoretical Basis}

In particular, we make use of theoretical models based in Regge's Theory and perturbative models of Quantum Cromodynamics (QCD). Furthermore, we consider the exchange Pomeron model, understood as virtual object that makes possible the exclusive (diffractive) production analysis; thus this is the focus of this work. The inclusive process production occurs in the following way: two hadrons (protons and/or nucleons) interact in collision, and this results in two quarks, a charm and an anti-charm. Then, we use a color evaporation model (CEM) in order to hadronize the two quarks to result in a quarkonium bound state. This model provides us a cross section for a process in which the partons (quarks and gluons) of two hadrons (protons and/or nucleons), namely $h_{1}$ and $h_{2}$, interact for producing a heavy state called quarkonium $H$ given by the cross section of pair production of heavy quarks, summed over all color states and spin, represented by $h_{1}+h_{2} \rightarrow H\left(n J^{C P}\right)+X$. All information on the non-perturbative transition of the pair $Q \bar{Q}$ for the heavy $H$ quarkonium with quantum numbers $J^{C P}$ is contained in the factor $F_{n J^{C P}}$ that a priori depends of all quantum numbers ${ }^{2,3}$

$$
\sigma\left(h_{1} h_{2} \rightarrow H\left[n J^{C P}\right] X\right)=F_{n J^{C P}} \bar{\sigma}\left(h_{1} h_{2} \rightarrow Q \bar{Q} X\right),
$$

where $\bar{\sigma}(Q \bar{Q} X)$ is the total cross section of open production of heavy quarks calculated by integration over the invariant mass of massive pair $Q \bar{Q}$ on $2 m_{Q}$ to $2 m_{0}$, being $2 m_{0}$ the associated mass of $D$ meson. This can be written as

$$
\sigma_{p p}\left(\sqrt{s}, m_{Q}^{2}\right)=\sum_{i, j=q, \bar{q}, g} \int d x_{1} d x_{2} f_{i}^{p}\left(x_{1}, \mu_{F}^{2}\right) f_{j}^{p}\left(x_{2}, \mu_{F}^{2}\right) \hat{\sigma}_{i j}\left(\sqrt{s}, m_{Q}^{2}, \mu_{F}^{2}, \mu_{R}^{2}\right),
$$

where $x_{1}$ and $x_{2}$ are the momentum fraction due to partonic collision, $f_{i}^{p}$ is the parton distribution of protons (Parton Distribution Function); we assume also that the factorization and renormalization scale are identical, $\mu=\mu_{F}=\mu_{R}$. In this work we use $\mu=2 m_{Q}$, setting the quark mass equal to $1.4 \mathrm{GeV}$. Such parameters give us a reasonable description of open production of heavy flavors ${ }^{4-6}$. The factor $F_{n J^{C P}}$ is determined experimentally ${ }^{7}$ being equal to $F_{11} \approx 2.5 \times 10^{-2}$. Now, we analyze the diffractive exclusive production, modeled by a single exchange of pomeron. For this, we will consider the approach given by Ingelman-Schlein ${ }^{8}$, where the pomeron structure (quark and gluon content) is explored. In the case of single diffraction, a pomeron is emitted by one of the hadrons. This hadron is detected in principle, in the final state and the remaining hadrons spread in relation to the emitted pomeron. The diffractive cross section of one hadron-hadron collision is factorized as product of pomeron-hadron cross section and Pomeron flux factor ${ }^{8}$. Explicitly, the pomeron-hadron cross section is written as

$$
\begin{aligned}
\frac{d \sigma(P+h \rightarrow Q \bar{Q}+X)}{d x_{1} d x_{2}}= & \sum_{i, j=q, \bar{q}, g} \frac{f_{i / P}\left(x_{1} / x_{P}^{(1)}, \mu_{F}^{2}\right)}{x_{P}^{(1)}} \\
& \times f_{i / h_{2}}\left(x_{2}, \mu_{F}^{2}\right) \hat{\sigma}\left(\sqrt{s}, m_{Q}^{2}, \mu_{F}^{2}, \mu_{R}^{2}\right)+(1 \Leftrightarrow 2),
\end{aligned}
$$


where the pomeron kinetic variable $x_{P}$ is defined as $x_{P}^{(i)}=x_{P}^{(j)}$ and $\sqrt{s_{P}^{(j)}}$ is the centre of mass energy of jth pomeron-hadron system and $\sqrt{s_{i j}}=\sqrt{s}$ is the centre of mass energy of ith hadron-hadron. The flux factor gives the emission rate of pomeron by the hadron, here we use the experimental analysis of the diffractive structure function ${ }^{9}$, where the dependency on $x_{P}$ is parameterized using flux factor motivated by the theory of Regge. In addiction, in Eq. (3) the partonic distribution function $f_{i / h_{2}}\left(x_{2}, \mu_{F}^{2}\right)$, that in the numeric calculations was used the DPDF's (Diffractive Partonic Distribution Function) from the collaboration H1 FPS ${ }^{9-11}$ for PDF's. We must also include corrections of multiple-pomerons exchange. In this calculation, we will consider the modification of the cross section by scattering effects of multiple pomerons due to the hadrons involved, a proton and a lead nucleus $(\mathrm{Pb})$. This is taken into account through the gap survival probability factor, $\left\langle|S|^{2}\right\rangle=4.39$, which can be described in terms of screening corrections or absorption ${ }^{12-14}$. This suppression factor of a hard process accompanied by a rapidity gap does not only depend of probability of survival initial state, but it is also sensitive to the spatial distribution of partons within the input hadrons and so the dynamics of the entire diffractive part of the scattering matrix. The survival factor of large rapidity gap (LRG) on one hadronic final state is the probability of a given LGR not be filled in by sub-products, that originates from the soft rescattering of spectator partons and/or of gluons emitted by partons belonging to hard interaction.

\section{Results and Final Considerations}

The analyzes concluded that, with enough reliability, the theoretical prediction produced by numerical analysis agrees with the accelerators data including the LHC

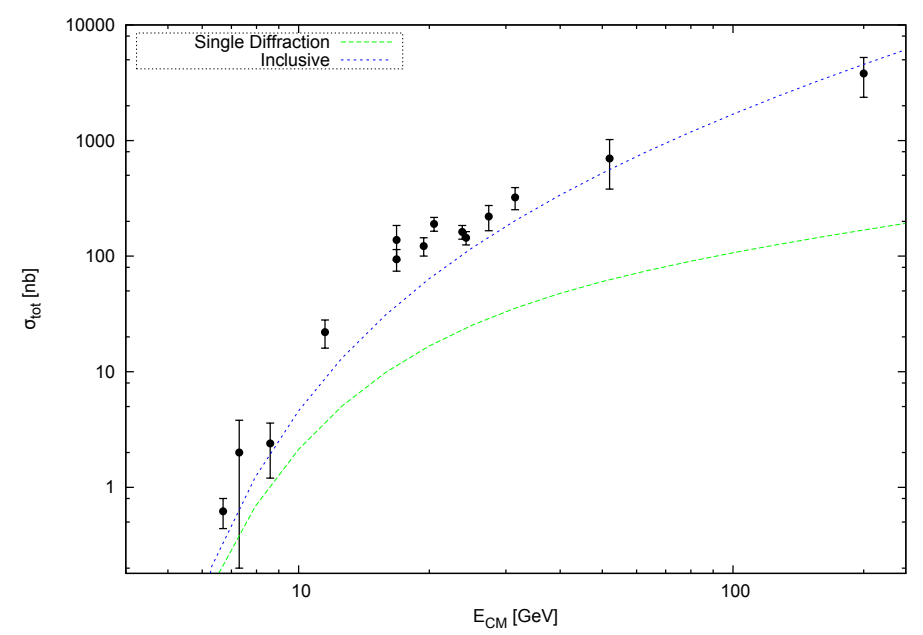

Fig. 1. Total cross section versus centre of mass energy for inclusive hadronic production (blue curve). The green curve represents the total cross section for diffractive production. 
data for the inclusive process. Fig. 1 displays a summary of the analysis. The experimental data on the inclusive production in the high energy regime are presented in their proper errors (up $200 \mathrm{GeV}$ regarding the RHIC accelerator). The blue line represents the theoretical prediction of the color evaporation model in function of the center of mass energy. The behavior and the order of magnitude are consistent with the data. In short, these promising results led us to develop predictions now for exclusive diffractive production processes of charmonium in $\mathrm{p}-\mathrm{Pb}$ collisions. The green line represents the total cross section of the single diffraction quarkonium $J / \psi$ production, which by the gap survival factor, allows estimate estimate the production rate for this process. We obtained the following predictions for the LHC energy regime: $852 \mathrm{nb}$ e $992 \mathrm{nb}$, for energies of $5.02 \mathrm{TeV}$ and $8.8 \mathrm{TeV}$ respectively. Here we use the gap survival factor given by Ref. ${ }^{12}$ and again, the partonic distributions of collaborations in Refs. ${ }^{9-11}$. That is, to obtain the predictions $p-A$, we multiply the result of the $p-p$ process by the respective gap survival factor (assuming very weak dependence of the inelastic cross section with energy).

\section{References}

1. M. V. T. Machado, Eur. Phys. J. C 54, 443 (2008).

2. J. F. Amundson, O. J. P. Eboli, E. M. Gregores, and F. Halzen, Phys. Lett. B390, 323 (1997).

3. C. Brenner Mariotto, M. B. Gay Ducati, and G. Ingelman, Eur. Phys. J. C 23, 527 (2002).

4. M. L. Mangano, P. Nason, and G. Ridolfi, Nucl. Phys. B373, 295 (1992).

5. A. Petrelli, M. Cacciari, M. Greco, F. Maltoni, and M. L. Mangano, Nucl. Phys. B514, 245 (1998).

6. S. Frixione et al., Adv. Ser. Direct High Energ. Phys. 15, 607 (1998).

7. G. A. Schuler and R. Vogt, Phys. Lett. B387, 181 (1996).

8. G. Ingelman and P. E. Schlein, Phys. Lett. B152, 256 (1985).

9. H1 Collaboration, A. Aktas et al., Eur. Phys. J C48, 715 (2006).

10. S. Atashbar Tehrani et al., arXiv:1109.0912v5 [hep-ph], (2011).

11. A. D. Martin et al., Phys. Lett. B604, 61 (2004).

12. R. Engel et al., Z.Phys. C74, 687 (1997).

13. J. D. Bjorken, Int. J. Mod. Phys. A7, 4189 (1992).

14. A. B. Kaidalov, V. A. Khoze, A. D. Martin, and M. G. Ryskin, Eur. Phys. J. C21, 521 (2001). 